\title{
National Research Council endorses genome project
}

\section{- Technology development essential - Initial work can start at once}

\section{Washington}

IF enthusiastic support from the National Research Council (NRC) counts for anything, the project to map and sequence the human genome has been given quite a boost. In a report released today, a committee of the NRC chaired by Bruce Alberts of the University of California at San Francisco says increased understanding of the human genome "merits a special effort that should be organized and funded specifically for this purpose".

A project to map and sequence the human genome is in some senses already a reality. The Department of Energy has been conducting research at three of its national laboratories aimed at starting the job (see Nature 331, 103; 1988), and the National Institutes of Health have received some $\$ 17.3$ million specifically for mapping, in addition to the estimated $\$ 200$ million it spends annually for genetics research relevant to a genome project. The Howard Hughes Medical Institute is also a substantial contributor to efforts to develop a genetic linkage map, and private companies such as Collaborative Research are also involved.

But a project aimed ultimately at sequencing all 3,000 million base pairs in the human genome has been regarded by most as beyond the resources of any individual company or federal agency. Critics have warned that such a 'big science' biology project would inevitably drain money from other areas of research.

The NRC report, although acknowledging that there are still organizational problems, argues that the scientific merits of the project outweigh its potential liabilities. It suggests that full-scale mapping should begin at once. This would include both genetic linkage mapping - describing the relative positions on different chromosomes of inherited traits - and physical mapping to ascribe absolute positions to cloned DNA fragments.

The report recommends against a large, central facility to begin sequencing the genome, until the technology needed for accurate, high-speed sequencing is improved. Instead, Alberts' panel suggests giving money to individual researchers and medium-sized multidisciplinary groups to achieve the necessary five-tenfold increases in scale and speed of sequencing. Peer review should be used to assess proposals for funding. The panel also suggests a pilot project to sequence 1 million continuous nucleotides, about 10 times more than accomplished so far.

Alberts says the committee is keen to emphasize that although the project's goal is to learn more about the human genome, such an understanding will require continued work on the genetics of other species as well. He points out that yeast cells have many of the same proteins as human cells, but the yeast cells provide a much simpler model for study.

The NRC report says that the genome project will be expensive. The panel estimates $\$ 200$ million will be needed annually for 15 years to complete the mapping and sequencing. This money "should not be diverted from the current federal research budget for biomedical sciences", but the report is unclear about what source can be tapped.

Both the federal government and Congress have been trying to decide how the genome project should be coordinated. At present there is an interagency committee chaired by the White House Office of Science and Technology Policy that has oversight responsibility for federal genome activities. But the influence this committee will have is still unclear. The NRC report concludes that a central organizing body will ultimately be needed for coordination.

But the committee could not reach a unanimous conclusion about who should be in charge. Most favour placing responsibility for the project within one federal agency - either the National Institutes of Health, the Department of Energy or the National Science Foundation. Other options considered are an inter-agency committee, or a separate administrative body. But for each of these proposals, Alberts says an independent, influential scientific advisory board is essential.

The initial stage of the genome project should focus primarily on development of appropriate technologies. Alberts says a lot of money for a genome project will allow researchers to take the time to work on initial be methodological issues.

Ultimately, of course, the project will develop mountains of genetic data. Although some have worried that these data may be used inappropriately to implement some social agenda, the report concludes that such information will be gathered anyway, and a coordinated and high quality effort will reduce "the chance of misuse of poorly organized information".
Move to ban sex determination

\section{Bangalore}

THE rural Indian state of Maharastra has decided to introduce legislation to ban the use of amniocentesis tests strictly for fetal sex determination. This practice, which is becoming prevalent all over India, has led to an alarming increase in abortions. An estimated 78,000 female fetuses were aborted in India between 1978 and 1982, a high figure in part attributable to the increasing number of private clinics that provide amniocentesis services.

According to D. T. Joseph, Secretary of the Maharastra Public Health Department, the ban will not affect the use of amniocentesis tests to detect genetic disorders such as Down's syndrome and spina bifida. But some doctors do not believe the ban will halt the growing number of amniocentesis-linked abortions, especially in those cultures where producing male progeny is considered essential.

Since 1975, the government of India has banned public hospitals from performing amniocentesis tests for the specific purpose of determining the sex of the fetus, but private clinics do not fall under this legislation. Although it is illegal to have an amniocentesis test performed with the intent of aborting a fetus if it is the 'wrong' sex, nobody has ever been prosecuted for breaking these laws. The cost for an amniocentesis test is about $70-600$ rupees $(£ 4-£ 35)$, and the cost for an abortion is about the same. The two together, many argue, are much cheaper than the cost of marrying off a daughter.

P. Phatnani, a forensic medicine expert, feels that a ban on amniocentesis tests will not solve this growing problem. He believes that a social awareness of the abuse of the technique is needed. Others feel that such tests should only be allowed in hospitals, and only when there is a family history of genetic birth defects. Medicolegal experts suggest that these tests be carried out exclusively by practitioners specifically licensed to administer the test to ensure there is no abuse.

But many doctors who work in amniocentesis testing centres support the practice, believing that a couple should be able to choose the sex of their children, and that any woman who wants the technique performed will get it done, even by an unqualified person. Others support the belief that by allowing this procedure, they are helping to keep an overflowing population in check, even though recent studies prove that this is not the case. Despite the fact that India has had an adverse sex ratio for several decades now, 935 women to 1,000 men, the population continues to grow. Radhakrishna Rao 